FR-PHENO-2011-006.

\title{
New spectra in the HEIDI Higgs models
}

\author{
J. J. van der Bij and B. Puliçe \\ Institut für Physik, Albert-Ludwigs Universität Freiburg \\ H. Herderstr. 3, 79104 Freiburg i.B., Germany
}

\begin{abstract}
We study the so-called HEIDI models, which are renormalizable extensions of the standard model with a higher dimensional scalar singlet field. As an additional parameter we consider a higher-dimensional mixing mass parameter. This leads to enriched possibilities compared to a previous study. We find effective spectral densities of the Higgs propagator, consisting of one, two or no particle peaks, together with a continuum. We compare with the LEP-2 data and determine for which range of the model parameters the data can be described. Assuming two peaks to be present we find for the new mass scale $\nu \approx 56 \pm 12 \mathrm{GeV}$, largely independent of the dimension. In the limiting case of $d \rightarrow 6$ and two peaks we find a higher dimensional coupling constant $\alpha_{6}=0.70 \pm 0.18$, indicative of strong interactions among the higher dimensional fields. The LHC will not be able to study this Higgs field.
\end{abstract}




\section{Introduction.}

With the developments from high energy colliders like LEP and the Tevatron the standard model (SM) has been established up to the loop level. The main missing ingredient is the direct detection of the SM Higgs boson. The four LEP experiments ALEPH, DELPHI, L3 and OPAL have extensively searched for the Higgs boson. The final combined result has been published in [1. The absence of a clear signal has led to a lower limit on the Higgs boson mass of $114.4 \mathrm{GeV}$ at the $95 \%$ confidence level.

Although no clear signal was found the data have some intriguing features, that can be interpreted as evidence for Higgs bosons beyond the standard model. There are excesses at $98 \mathrm{GeV}$ and $115 \mathrm{GeV}$, that were interpreted in [2-5] as evidence for the presence of two Higgs bosons. However the fits are somewhat unsatisfactory. Actually the precision measurements leave only very little space for extensions to the SM, as these tend to spoil the agreement with experiment due to a variety of effects, one of the most important of which is the appearance of flavor-changing neutral currents. For instance supersymmetric models have to finely tune a number of parameters. This leaves only one type of extensions that are safe, namely the singlet extensions. Experimentally right handed neutrino's appear to exist. Since these are singlets a natural extension of the SM is the existence of singlet scalars too. These will only have a very limited effect on radiative corrections, since they appear only in two-loop calculations. The simplest model giving rise to two Higgs bosons is the Hill model [6]. For a mini-review on singlet extensions see [7].

Of great interest are renormalizable extensions with a higher-dimensional singlet scalar. In such models the Higgs propagator can change significantly from its standard model form, in particular if the higher dimensions are non-compact. In this case the Higgs can become very broad, even though it is light and weakly interacting, while the spectrum is not anymore a single particle, but at least partly a continuum. Such a Higgs boson cannot be seen at the LHC, because for a light Higgs boson one needs a narrow peak to have a signal at the LHC. It is for this reason that we call these models HEIDI models, for hidi(ng) in high-D(imensions), in analogy to SUSY for supersymmetry. In a previous paper [8] these models were compared with the LEP-2 data and it was shown that a good fit was possible, though in a somewhat narrow range of parameters. In this paper we extend the study of [8], by including an extra parameter, a mass term mixing the higher dimensional fields among each other. This extra term would be generated by renormalization effects in any case and should therefore be included when comparing with the data. The analysis is straightforward, but gives a number of extra possibilities, i.e. instead of only one peak plus a continuum one finds the possibility of having two peaks or even no peak at all in the Higgs propagator.

The paper is organized as follows. In section 2 we describe the model and the Higgs 
propagator. In section 3 we describe the possibilities for the Källén-Lehmann spectral density. In section 4 we compare with the LEP-2 data. In section 5 we discuss the results.

\section{The model and the Higgs propagator.}

The model consists of the standard model with the Higgs field $\Phi$ and in addition a scalar field $H$, moving in $d=4+\gamma$ dimensions. Normally speaking this would lead to a nonrenormalizable theory. However since the only interaction is of the form $H \Phi^{\dagger} \Phi$, which is superrenormalizable in four dimensions, the theory stays renormalizable. An analysis of the power counting of divergences shows that one can associate the canonical dimension $1+\gamma / 2$ to the $H$-field. This means that the theory stays renormalizable as long as $\gamma \leq 2$. When one assumes, that the extra dimensions are compact, for instance a torus, with radius $R=L / 2 \pi$, one can simply expand the $d$-dimensional field $H(x)$ in terms of Fourier modes:

$$
H(x)=\frac{1}{\sqrt{2} L^{\gamma / 2}} \sum_{\vec{k}} H_{\vec{k}}\left(x_{\mu}\right) e^{i \frac{2 \pi}{L} \vec{k} \vec{x}}, \quad H_{\vec{k}}=H_{-\vec{k}}^{*}
$$

Here $x_{\mu}$ is a four-vector, $\vec{x}$ is $\gamma$-dimensional and the $\gamma$ components of $\vec{k}$ take only integer values.

In terms of the modes $H_{i}$ the Lagrangian, that we use, is the following:

$$
\begin{aligned}
L & =-\frac{1}{2} D_{\mu} \Phi^{\dagger} D_{\mu} \Phi-\frac{1}{2} \sum\left(\partial_{\mu} H_{k}\right)^{2}+\frac{M_{0}^{2}}{4} \Phi^{\dagger} \Phi-\frac{\lambda}{8}\left(\Phi^{\dagger} \Phi\right)^{2}-\sum \frac{m_{k}^{2}}{2} H_{k}^{2} \\
& -\frac{g}{2} \Phi^{\dagger} \Phi \sum H_{k}-\frac{\zeta}{2} \sum H_{i} H_{j}
\end{aligned}
$$

Here $m_{k}^{2}=m^{2}+m_{\gamma}^{2} \vec{k}^{2}$, where $\vec{k}$ is a $\gamma$-dimensional vector, $m_{\gamma}=2 \pi / L$ and $m$ is a $d$-dimensional mass term for the field $H$.

This Lagrangian differs from the one in [8] through the presence of the $\sum H_{i} H_{j}$ term. This term however would be present in general, while it is generated via loop effects in the propagator through the $\Phi^{\dagger} \Phi \sum H_{k}$ interactions. The last two terms can be written as so-called brane-bulk terms of the form:

$$
S=\int d^{4+\gamma} x \prod_{i=1}^{\gamma} \delta\left(x_{4+i}\right)\left(g_{B} H(x) \Phi^{\dagger} \Phi-\zeta_{B} H(x) H(x)\right)
$$

It is now straightforward to follow the steps of $[8]$. One expands around the minimum of the potential and inverts the quadratic part of the Lagrangian, in order to get the propagator. 
Introducing the parameter $M^{2}=\lambda v^{2}$, where $v$ is the weak scale, the quadratic piece takes the form:

$$
P_{i j}^{-1}\left(q^{2}\right)=\left(\begin{array}{ccccc}
q^{2}+M^{2} & -g v & -g v & \cdots & -g v \\
-g v & q^{2}+m_{1}^{2}+\zeta & \zeta & \cdots & \zeta \\
-g v & \zeta & q^{2}+m_{2}^{2}+\zeta & & \zeta \\
\vdots & \vdots & & \ddots & \vdots \\
-g v & \zeta & \zeta & \cdots & q^{2}+m_{n}^{2}+\zeta
\end{array}\right)
$$

We need to find the Higgs-field propagator which will be the $(1,1)$ component of the matrix $P_{i j}$. For this reason, we use the following determinant formulas:

$$
\left|\begin{array}{ccccc}
a_{1}+\alpha & \alpha & \alpha & \cdots & \alpha \\
\alpha & a_{2}+\alpha & \alpha & \cdots & \alpha \\
\alpha & \alpha & a_{3}+\alpha & & \alpha \\
\vdots & \vdots & & \ddots & \vdots \\
\alpha & \alpha & \alpha & \cdots & a_{n}+\alpha
\end{array}\right|=\prod a_{n}\left(1+\alpha \sum \frac{1}{a_{n}}\right)
$$

and

$$
\left|\begin{array}{ccccc}
A & \beta & \beta & \cdots & \beta \\
\beta & a_{1}+\alpha & \alpha & \cdots & \alpha \\
\beta & \alpha & a_{2}+\alpha & & \alpha \\
\vdots & \vdots & & \ddots & \vdots \\
\beta & \alpha & \alpha & \cdots & a_{n}+\alpha
\end{array}\right|=A \prod a_{n}\left(1-\frac{\beta^{2}}{A} \sum \frac{1}{a_{n}}+\alpha \sum \frac{1}{a_{n}}\right)
$$

where the symbols correspond to the following expressions

$$
\begin{aligned}
A & =q^{2}+M^{2} \\
\beta & =-g v \\
a_{n} & =q^{2}+m_{n}^{2} \\
\alpha & =\zeta
\end{aligned}
$$

The ratio of (5) to (6) gives us the $(1,1)$ component of the inverse of the matrix (44), i.e. the Higgs-field propagator

$$
D_{H H}\left(q^{2}\right)=\frac{1+\zeta \frac{\Gamma(1-\gamma / 2)}{(4 \pi)^{\gamma / 2}}\left(q^{2}+m^{2}\right)^{\frac{\gamma-2}{2}}}{\left(q^{2}+M^{2}\right)\left(1-\frac{\Gamma(1-\gamma / 2)}{(4 \pi)^{\gamma / 2}}\left(q^{2}+m^{2}\right)^{\frac{\gamma-2}{2}}\left(\frac{g^{2} v^{2}}{\left(q^{2}+M^{2}\right)}-\zeta\right)\right)}
$$

where we have taken the continuum limit

$$
\sum \frac{1}{a_{n}}=\frac{\Gamma(1-\gamma / 2)}{(4 \pi)^{\gamma / 2}}\left(q^{2}+m^{2}\right)^{\frac{\gamma-2}{2}}
$$


We make the following definitions:

$$
\begin{aligned}
& \mu^{8-d}=g^{2} v^{2} \frac{\Gamma(1-\gamma / 2)}{(4 \pi)^{\gamma / 2}} \\
& \nu^{6-d}=|\zeta| \frac{\Gamma(1-\gamma / 2)}{(4 \pi)^{\gamma / 2}}
\end{aligned}
$$

The Higgs-field propagator (8) becomes

$$
D_{H H}\left(q^{2}\right)=\left(q^{2}+M^{2}-\frac{\mu^{8-d}}{\left(q^{2}+m^{2}\right)^{\frac{6-d}{2}} \pm \nu^{6-d}}\right)^{-1}
$$

where the sign in front of the $\nu$ term is the sign of $\zeta$. In the rest of the paper $\nu$ is supposed to be a positive number. In the following we will use the auxiliary quantity $\delta=(6-d) / 2$.

\section{The Källén-Lehmann spectral density.}

Given the form of the Higgs-propagator found in the previous section one can derive from it the Källén-Lehmann [9, 10] spectral density. The spectral density $\rho(s)$ is simply given by the imaginary part of the propagator.

$$
\rho_{\mathrm{KL}}(s)=-\frac{1}{2 \pi i}(D(s+i \epsilon)-D(s-i \epsilon))
$$

In case there is a zero in the inverse propagator one has a pole, that shows up as a deltafunction in the Källén-Lehmann density. This is the case one is used to in ordinary field theory where the pole corresponds to a particle on the mass-shell. In the HEIDI models the spectrum is more general containing poles and a continuum. Different possibilities arise for different values of the parameters $m, M, \mu, \nu$ from (11). However not all possibilities correspond to a physically allowed spectral density. One must demand that there are no tachyon poles in the propagator. Such a pole corresponds to an instability in the theory, that arises when one does not expand around a minimum of the potential. Typically the potential would not be bounded from below. Alternatively the attractive force among the Higgs particles due to the high-D particles is larger than the repulsion from the selfcoupling [8]. Different possibilities are discussed in the subsections below. The range $4<d<6$ can be treated as a whole. The limiting case $d \rightarrow 6$ is treated separately. The continuum part $\rho_{c}(s)$ of the spectral density for $4<d<6$ is given by

$$
\rho_{c}(s)=\frac{1}{\pi} \frac{\mu^{8-d}\left(s-m^{2}\right)^{\delta} \sin (\pi \delta) \theta\left(s-m^{2}\right)}{\left\{\left(M^{2}-s\right)\left[\left(s-m^{2}\right)^{\delta} \cos (\pi \delta) \pm \nu^{2 \delta}\right]-\mu^{8-d}\right\}^{2}+\sin ^{2}(\pi \delta)\left(M^{2}-s\right)^{2}\left(s-m^{2}\right)^{2 \delta}}
$$




\subsection{Negative sign in front of the $\nu$ term.}

In the case the $\nu$ term appears with a negative sign, the condition for the absence of a tachyon pole is:

No tachyon: $M^{2}\left(m^{6-d}-\nu^{6-d}\right)>\mu^{8-d}$.

Assuming the tachyon condition is fulfilled we consider several cases.

$M^{2}>m^{2}$ : there is one pole in the propagator.

$M^{2}<m^{2}$ : there is one pole in the propagator if: $\nu^{6-d}\left(m^{2}-M^{2}\right)<\mu^{8-d}$.

$M^{2}<m^{2}$ : there are two poles in the propagator if: $\nu^{6-d}\left(m^{2}-M^{2}\right)>\mu^{8-d}$.

\subsection{The case $\nu=0$.}

The case $\nu=0$ was dicussed in 8 .

The no-tachyon condition reads: $M^{2} m^{6-d}>\mu^{8-d}$.

In this case there is always one pole plus a continuum.

\subsection{Positive sign in front of the $\nu$ term.}

In the case of a positive sign in front of the $\nu$ term the conditions are the following:

No tachyon: $M^{2}\left(m^{6-d}+\nu^{6-d}\right)>\mu^{8-d}$.

$M^{2}>m^{2}$ : no pole if: $\nu^{6-d}\left(M^{2}-m^{2}\right)>\mu^{8-d}$.

$M^{2}>m^{2}$ : one pole if: $\nu^{6-d}\left(M^{2}-m^{2}\right)<\mu^{8-d}$.

$M^{2}<m^{2}$ : always one pole.

\subsection{1 $m=0$.}

In the theory we have four mass parameters $m, M, \mu, \nu$. This makes for a fairly complicated analysis and one might wonder whether one could eliminate one or more of the parameters, without getting into trouble with renormalizability. Of course one could put $\mu=0$, but 
this simply decouples the higher dimensions from the four dimensional fields and one gets the standard model back. More interesting is to put $m=0$. This is actually consistent with renormalization, as the higher dimensional mass term does not get generated through the interactions with the ordinary Higgs field. Only the $\nu$-term is genererated by loop effects. In the case that $\nu>0$ one can indeed put $m=0$, without getting a tachyon in the propagator. The no tachyon condition becomes: $M^{2} \nu^{6-d}>\mu^{8-d}$. This implies that there is no pole in the propagator and one has a continuum only. The situation is somewhat similar to the ideas in [11, where the singlet fields are to be conformal invariant, however the conformal invariance is here broken by the $\mu, \nu$ and $M$ terms. This is necessary in order to get a satisfactory spectrum [12].

In discussions the question often arises, whether putting a parameter like $m$ equal to zero, should be considered an unnatural fine-tuning, since this relation is not due to a symmetry. It must be emphasized, that the condition is preserved under renormalization. Therefore one cannot say that imposing $m=0$ is really arbitrary, as long as one has no deeper insight in some form of underlying dynamics. The question is somewhat philosophical: should one only consider the terms that are needed for renormalizability or assume all terms to be present that are allowed by renormalizability? In most ordinary models these classes are the same, so the question does not arise.

\subsection{The case $d \rightarrow 6$.}

For the critical dimension $d=6$ one cannot simply copy the formulas from section 2 . In $d=6$ the $\Gamma$-function develops a pole, so its value cannot be absorbed in the definition of the mass parameters, that end up in the Higgs propagator. Instead one has to use a limiting procedure. Another way to see the problem is by noting that the parameter $\nu$ becomes dimensionles and is therefore to be considered as a coupling constant and not as a mass parameter. It was noted before [13 16 , that in $d=6$ the $\nu$ parameter has a renormalization group running already at the tree level. There are different ways to write the propagator in this case, depending on how one defines the new parameters in the propagator. We choose a new variable $\alpha_{6}$ to describe the propagator. The name is chosen to make clear that we are talking about a coupling constant. In the formulas $\alpha_{6}$ can be taken to be positive, negative or zero, zero corresponding to the case without $\nu$ term. The form of the Higgs propagator becomes

$$
D_{H H}\left(q^{2}\right)=\left(q^{2}+M^{2}+\mu^{2} \frac{\log \left(\left(q^{2}+m^{2}\right) / m^{2}\right)}{1+\alpha_{6} \log \left(\left(q^{2}+m^{2}\right) / m^{2}\right)}\right)^{-1}
$$

In this form $\alpha_{6}=0$ reproduces the propagator from [8]. The analysis is simple. The 
absence of a tachyon requires

$$
\alpha_{6} \geq 0 \text { and } M^{2} \geq 0
$$

Furthermore one has

$\alpha_{6}\left(m^{2}-M^{2}\right)<\mu^{2}:$ there is one pole in the propagator.

$\alpha_{6}\left(m^{2}-M^{2}\right) \geq \mu^{2}:$ there are two poles in the propagator.

Because of the possibility of having two poles the limit $d \rightarrow 6$ is similar to the $\nu<0$ case in the range $4<d<6$. Putting $m^{2}=0$ in the $q^{2}+m^{2}$ term would lead to a tachyon in the propagator.

The continuum spectral density $\rho_{c}(s)$ is here given by:

$$
\rho_{c}(s)=\frac{\mu^{2} \theta\left(s-m^{2}\right)}{\left[-s+M^{2}+\left(\mu^{2}-\alpha_{6} s+\alpha_{6} M^{2}\right) \log \left(\left(m^{2}-s\right) / m^{2}\right)\right]^{2}+\pi^{2}\left(\mu^{2}-\alpha_{6} s+\alpha_{6} M^{2}\right)^{2}}
$$

\section{Comparison with the LEP-2 data}

\subsection{Description of the data}

As mentioned in the introduction the LEP-2 Higgs search data have some features, that make them interpretable within our model. There is a $2.3 \sigma$ effect seen by all experiments at around $98 \mathrm{GeV}$. A somewhat less significant $1.7 \sigma$ excess is seen around $115 \mathrm{GeV}$. Finally over the whole range $\sqrt{s}>100 \mathrm{GeV}$ the confidence level is less than expected from background. Within the minimal supersymmetric standard model (MSSM) and other extensions [2-5], the excesses at $98 \mathrm{GeV}$ and $115 \mathrm{GeV}$ were interpreted as evidence for the presence of two Higgs bosons $H_{i}$ with couplings to matter reduced by a factor $\alpha_{i}$, giving $g_{i}^{2}=\alpha_{i} g_{S M-H i g g s}^{2}$. We will call such Higgs bosons fractional Higgses in the following. The excess at $98 \mathrm{GeV}$ is well described by a $10 \%$ fractional Higgs. More precisely [3] gives limits $0.056<\alpha_{-}<0.114$ and a mass range $95 \mathrm{GeV}<m_{\text {Higgs }}<101 \mathrm{GeV}$. The second peak at $115 \mathrm{GeV}$ is then interpreted as a second Higgs boson with $\alpha_{+}=0.9$. The first peak at 98 $\mathrm{GeV}$ is rather convincing. The second one at $115 \mathrm{GeV}$ is compatible with the data, but not really preferred as the data at $115 \mathrm{GeV}$ are also compatible with pure background with a similar confidence level. Actually the data correspond roughly to background plus one half of a Higgs boson, however with a large uncertainty.

Within our model there are different possibilities for the spectrum. One can have one, two or no peaks plus a continuum in the spectrum. We propose different ways to analyze 
the data for the different cases. In the case of two peaks we have, for a fixed dimension $d$, four parameters $M, m, \mu, \nu$ and can use the strength and location of the two peaks to completely fix these parameters. This can be straightforwardly done. Our model has the advantage over the MSSM, that the sum of the strenghts of the peaks does not have to be one, allowing for more freedom. Hereby we ignore the possibility of a continuum between the peaks, which is not very pronounced in the data.

Another way to analyze the data was presented in [8]. If there is one peak plus a continuum one can do the following. One takes the excess at $98 \mathrm{GeV}$ at face value and interprets it as the delta-peak in the propagator. The excess at $115 \mathrm{GeV}$ is interpreted as an enhancement due to the continuum of the Higgs propagator. Because of the uncertainty of this excess we will only demand that a Higgs integrated spectral density $\int \rho(s) d s>30 \%$ is present in the range $110 \mathrm{GeV}<\sqrt{s}<120 \mathrm{GeV}$.

In the case of no peaks one could take the special case $m=0$, allowing for a spectrum extending down to $m=0$. One could then worry, whether a possible continuum Higgs propagator has simply been overlooked, because the density is too low for the sensitivity of the LEP-2 experiments. One might hope to get a better fit to the LEP-1 indirect determination of the Higgs mass, which is quite low, about $89 \mathrm{GeV}$. However the published data have not been analyzed with this point of view in mind. There are limits in the literature on the strength of a single Higgs at every mass [1. However these cannot be used to analyze our model, as the size of the different mass bins is not given, so there is no way to scale the mass density. A precise comparison would need a complete reanalysis of the data. So we will not pursue this possibility here any further. Also the analyses with one or two peaks suffer from the fact that the data are not very precise and have not been analyzed with this type of model in mind. In particular the statistical significance of the deviations of the standard model is hard to determine. Rough estimates give about $3.0-3.4 \sigma$.

\subsection{Fit to the two-pole models.}

\subsubsection{Parameters and data.}

When one tries to fit the parameters to the LEP-2 data, one finds that both $M$ and $m$ are quite close to the location of the highest peak. It is therefore useful to define some auxiliary quantities.

We define:

$$
\begin{aligned}
m^{2} & =s_{+}+\epsilon\left(s_{+}-s_{-}\right) \\
M^{2} & =s_{+}-\Delta\left(s_{+}-s_{-}\right)
\end{aligned}
$$


Here $s_{+}$is the location of the most massive peak, with a contribution $\alpha_{+}$in the spectral density; $s_{-}$is the location of the less massive peak, with a contribution $\alpha_{-}$in the spectral density.

For a fixed dimension we have four data points to fit four parameters, that are therefore completely fixed. We vary the input values over the following range:

$$
\begin{aligned}
0.056 & <\alpha_{-} \quad<0.144 \\
0.3 & <\alpha_{+}<0.7 \\
95 \mathrm{GeV} & <\sqrt{s_{-}}<101 \mathrm{GeV} \\
111 \mathrm{GeV} & <\sqrt{s_{+}}<117 \mathrm{GeV}
\end{aligned}
$$

\subsubsection{Solution in the range $4<d<6$.}

One first finds $\epsilon$ from the equation:

$$
\delta^{2} \cdot \frac{\alpha_{-}}{1-\alpha_{-}} \cdot \frac{\alpha_{+}}{1-\alpha_{+}}=\epsilon(1+\epsilon)\left[\left(\frac{1+\epsilon}{\epsilon}\right)^{\delta / 2}-\left(\frac{\epsilon}{1+\epsilon}\right)^{\delta / 2}\right]^{2}
$$

For finite $\delta$, away from $d=6$ and small $\epsilon$ a good approximation $\epsilon_{\text {app }}$ to the solution is:

$$
\epsilon_{\text {app }}=\left(\delta^{2} \cdot \frac{\alpha_{-}}{1-\alpha_{-}} \cdot \frac{\alpha_{+}}{1-\alpha_{+}}\right)^{\frac{2}{d-4}}
$$

Next one can determine $\Delta$ from the equation:

$$
\frac{\alpha_{+}}{1-\alpha_{+}} \cdot \frac{1-\alpha_{-}}{\alpha_{-}} \cdot\left(\frac{1+\epsilon}{\epsilon}\right)^{\frac{d-4}{2}}=\left(\frac{1-\Delta}{\Delta}\right)^{2}
$$

Next one has :

$$
\mu^{8-d}=\Delta(1-\Delta)\left(s_{+}-s_{-}\right)^{1+\delta}\left((1+\epsilon)^{\delta}-\epsilon^{\delta}\right)
$$

and finally:

$$
\nu^{6-d}=\left(m^{2}-s_{-}\right)^{\delta}-\frac{\mu^{8-d}}{M^{2}-s_{-}}=\left(m^{2}-s_{+}\right)^{\delta}-\frac{\mu^{8-d}}{M^{2}-s_{+}}
$$


Trying to fit to the LEP-2 data we have small values for $\alpha_{-}$and $\left(s_{+}-s_{-}\right) / s_{+}$. One can take $\epsilon \approx 0$ and find an approximate solution:

$$
\begin{aligned}
\Delta & =\frac{\delta . \alpha_{-}}{1-\alpha_{-}+\delta \cdot \alpha_{-}} \\
\mu & =[\Delta(1-\Delta)]^{\frac{1}{8-d}} \sqrt{s_{+}-s_{-}} \\
\nu & =[1-\Delta]^{\frac{1}{6-d}} \sqrt{s_{+}-s_{-}}
\end{aligned}
$$

As also $\Delta$ is small the scale of $\nu$ is basically fixed independent of the dimension to about:

$$
\nu=56 \pm 12 \mathrm{GeV}
$$

This approximation works very well as long as one is not close to the limit $d \rightarrow 6$. In the case $d=4$ one reverts to the original Hill model, that has two peaks and no continuum. As in this case there is only one singlet mode, the presence or absence of the $\nu$ term makes no difference.

\subsubsection{Solution for the limit $d \rightarrow 6$.}

In the limiting case $d \rightarrow 6$ the term on the right side of equation 18 behaves like $\epsilon \log ^{2}(\epsilon)$. In this case one should not use the approximation $\epsilon \rightarrow 0$, but use the exact equations.

One first finds $\epsilon$ from the equation:

$$
\frac{\alpha_{-}}{1-\alpha_{-}} \cdot \frac{\alpha_{+}}{1-\alpha_{+}}=\epsilon(1+\epsilon) \log ^{2}\left(\frac{\epsilon}{1+\epsilon}\right)
$$

Next one can determine $\Delta$ from the equation:

$$
\frac{\alpha_{+}}{1-\alpha_{+}} \cdot \frac{1-\alpha_{-}}{\alpha_{-}} \cdot \frac{1+\epsilon}{\epsilon}=\left(\frac{1-\Delta}{\Delta}\right)^{2}\left(\frac{\log (\epsilon)+\log \left(\left(s_{+}-s_{-}\right) / m^{2}\right)}{\log (1+\epsilon)+\log \left(\left(s_{+}-s_{-}\right) / m^{2}\right)}\right)^{2}
$$

Next one has :

$$
\mu^{2}=\Delta(1-\Delta)\left(s_{+}-s_{-}\right)\left(\frac{1}{\log \left(\left(m^{2}-s_{+}\right) / m^{2}\right)}-\frac{1}{\log \left(\left(m^{2}-s_{-}\right) / m^{2}\right)}\right)
$$

and finally:

$$
\alpha_{6}=-\frac{\mu^{2}}{M^{2}-s_{-}}-\frac{1}{\log \left(\left(m^{2}-s_{-}\right) / m^{2}\right)}=-\frac{\mu^{2}}{M^{2}-s_{+}}-\frac{1}{\log \left(\left(m^{2}-s_{+}\right) / m^{2}\right)}
$$


We use these formulas to determine the values of $m, M, \mu, \alpha_{6}$ from the location and the strengths of the peaks. The values of $m$ and $M$ are always close to $\sqrt{s_{+}}$. For the range of $\mu$ we find $6 \mathrm{GeV}<\mu<22 \mathrm{GeV}$. For $\alpha_{6}$ we find $0.52<\alpha_{6}<0.88$. In particular the last number is interesting. It would be the first measurement of a higher-dimensional coupling constant. The value indicates a rather strong coupling.

\subsection{Models with one pole and a continuum.}

\subsubsection{Fitting the data.}

In this case we follow the procedure from [8]. We will take the excess at $98 \mathrm{GeV}$ at face value and interpret it as the delta-peak in the propagator. The excess at $115 \mathrm{GeV}$ is interpreted as an enhancement due to the continuum of the Higgs propagator. Because of the uncertainty of this excess we will only demand that a Higgs integrated spectral density $\int \rho(s) d s>30 \%$ is present in the range $110 \mathrm{GeV}<s^{1 / 2}<120 \mathrm{GeV}$. The delta-peak will be assumed to correspond to the peak at $98 \mathrm{GeV}$, with a fixed value of $\alpha_{-}$. Ultimately we will vary the location of the peak between $95 \mathrm{GeV}<\sqrt{s_{-}}<101 \mathrm{GeV}$ and the strength of the peak between $0.056<\alpha_{-}<0.144$. After fixing $\alpha_{-}$and $m_{-}$we have two free variables, which we take to be $\mu$ and $\nu$. If we also assume a value for $\mu$ and for $\nu$ all parameters and thereby the spectral density is known. We can then numerically integrate the spectral density over selected ranges of $s$. The allowed range of $\mu$ and $\nu$ is subsequently determined by the data at $115 \mathrm{GeV}$. Since the peak at $115 \mathrm{GeV}$ is not very well constrained, we demand here only that the integrated spectral density from $s_{\text {down }}=(110 \mathrm{GeV})^{2}$ to $s_{\text {up }}=(120 \mathrm{GeV})^{2}$ is larger than $30 \%$. In general the continuum starts very close to the lowest peak. This allows for a natural explanation, why the CL for the fit in the whole range from $100 \mathrm{GeV}$ to $110 \mathrm{GeV}$ is somewhat less than what is expected by pure background. The enhancement can be due to a slight, spread-out Higgs signal. Actually when fitting the data with the above conditions sometimes the integrated spectral density in the range $100 \mathrm{GeV}$ to 110 $\mathrm{GeV}$ can become rather large, which would lead to problems with the 95\% CL limits in this range. We therefore additionally demand that the integrated spectral density in this range is less than $30 \%$. There is no problem fitting the data with these conditions. As a final consistency check we have checked that the results are in agreement with the upper limit on the Higgs boson mass from precision measurements $m_{H}<158 \mathrm{GeV}$. The integrated spectral density above $158 \mathrm{GeV}$ is less than a few percent.

\subsubsection{Allowed range in the $\mu, \nu$ or $\mu, \alpha_{6}$ plane.}

Varying the input parameters as mentioned above, one determines for each dimension an allowed range in the $M, m, \mu, \nu$ hypervolume. Given the fact that one has four mass 


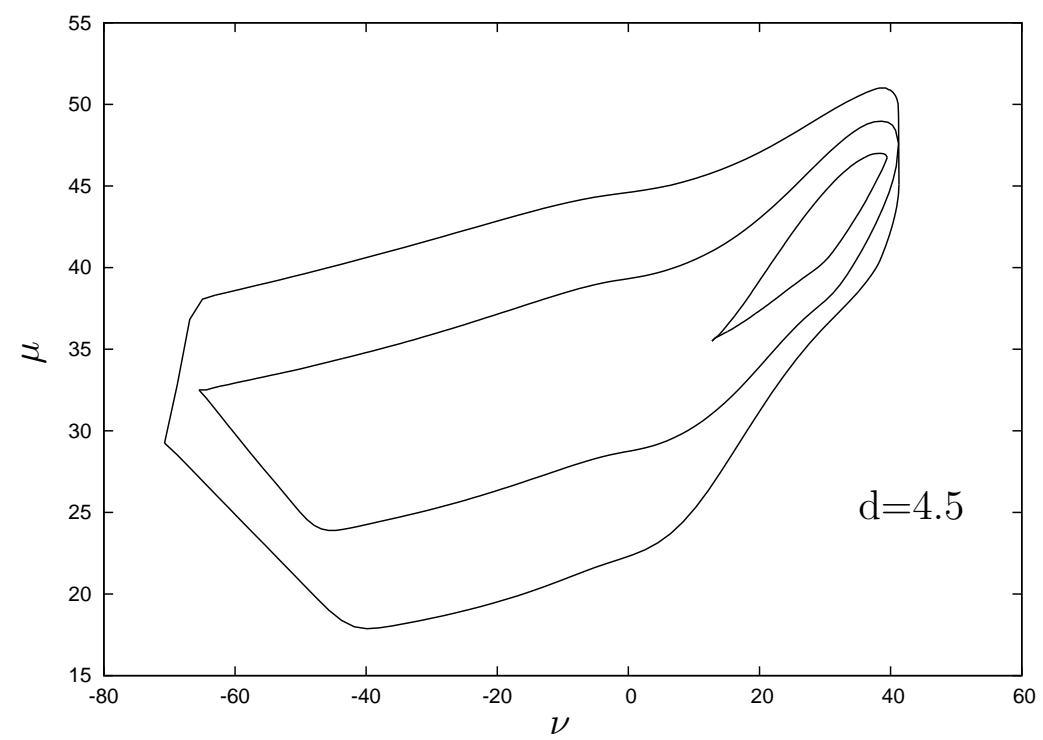

Figure 1: The allowed $\mu, \nu$-plane for the 4.5 dimensional HEIDI model.

parameters, one could make six different two-dimensional projections. However they are not equally interesting. The value of $m$ is always close to the location of the peak at $98 \mathrm{GeV}, M$ is in the neighbourhood of $115 \mathrm{GeV}$, but can move up to about $140 \mathrm{GeV}$. We therefore only give graphs of the projection in the $\mu, \nu$-plane which shows the most interesting behaviour.

The allowed range for the parameters $\mu$ and $\nu$ are given in the figures 1-3 for the dimensions $d=4.5,5.0,5.5$. In the figures three curves are drawn. The points within the outer curve are the points for which there is a solution within the range $0.056<\alpha_{-}<0.144$ and $95 \mathrm{GeV}<\sqrt{s_{-}}<101 \mathrm{GeV}$. For the points within the middle curve there is a solution with $\alpha_{-}=0.1$ and $\sqrt{s_{-}}=98 \mathrm{GeV}$. The points inside the inner curve have a solution for all values in the range $0.056<\alpha_{-}<0.144$ and $95 \mathrm{GeV}<\sqrt{s_{-}}<101 \mathrm{GeV}$.

For the case $d \rightarrow 6$ the procedure is the same, only the parameters are now $M, m, \mu, \alpha_{6}$. We give the projection on the $\mu, \alpha_{6}$ plane in figure 4 .

\section{Discussion}

We introduced an extra parameter $\nu$, a mass-mixing term for the higher dimensional fields, in the class of HEIDI models and have shown that this gives rise to a broader range of possibilities than was studied before. As a first conclusion, due to the extra parameter, we see that the spectra containing a single pole can describe the LEP-2 data in a rather 


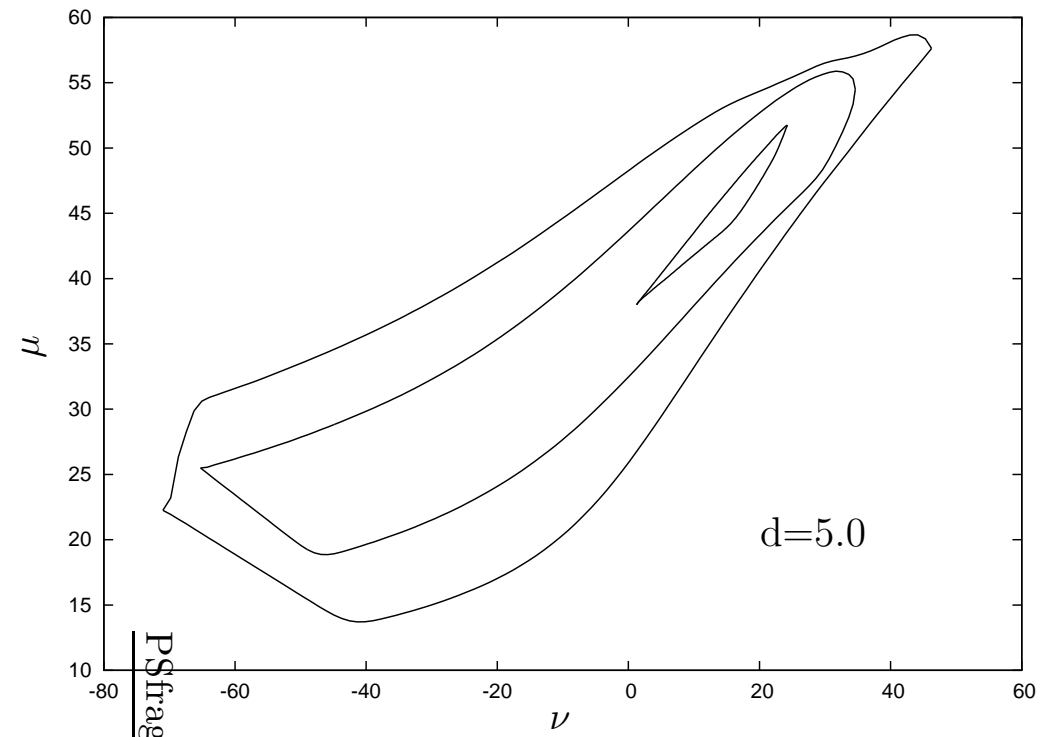

Figure 2: The allowed $\mu, \nu$-plane for the 5.0 dimensional HEIDI model.

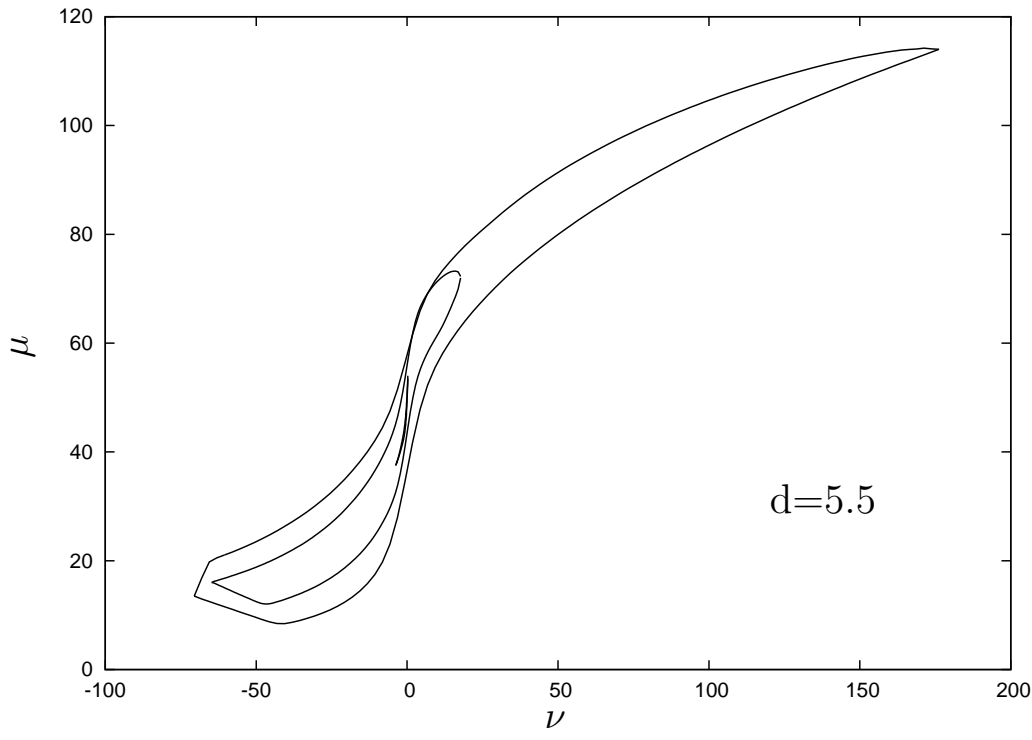

Figure 3: The allowed $\mu, \nu$-plane for the 5.5 dimensional HEIDI model. 


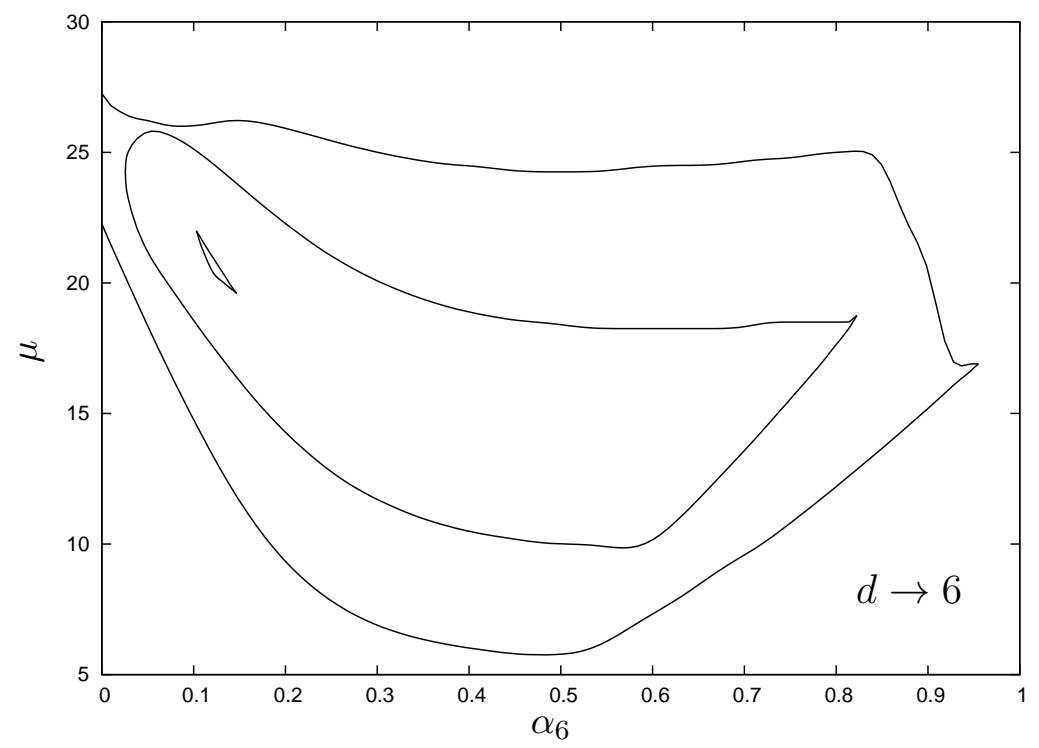

Figure 4: The allowed $\mu, \alpha_{6}$-plane for the 6 dimensional HEIDI model.

broad range of parameters, without any great form of fine-tuning, so these models are quite natural. One could even go as far as to say, that this should be the generic form of electroweak symmetry breaking. If the data are interpreted as having two peaks, it is interesting, that the new parameter can be determined rather precisely, largely independent of the dimension. Unfortunately the data are not very precise, otherwise one could have claimed the clear measurement of a new fundamental physical constant. It is deplorable, that LEP-2 was not run at a larger energy and a longer period. Clarity about this model can only come with a new lepton machine, the one most studied being the linear collider, which however might not be an optimal machine for this type of model, because of the beamstrahlung. However more detailed studies are needed here. From the theoretical point of view it is interesting, that the higher dimensional field could be massless without spoiling renormalizability, which would actually imply the absence of any particle pole.

As this type of models is rather unfamiliar one might be somewhat sceptical about their meaning. A typical objection is that we have never seen a fundamental field with a nontrivial Källén-Lehmann spectrum, so it would be unlikely that the Higgs-boson would be different. However one can easily make the objection, that we also have never seen a fundamental scalar field, so why should the Higgs field behave as other particles. The special place of the Higgs field in the theory is an argument in point of this view. Also the $\sigma$-resonance in the strong interactions looks quite similar to the Higgs field here.

One can of course take a more positive point of view. After all the original Higgs field was introduced to solve a fundamental problem. We know massive gauge vectorbosons exist, 
but by themselves they cannot have a unitary scattering matrix. In order to cure this problem a field with the same quantum numbers as the Higgs is needed. It cannot be too heavy, since that would violate unitarity. Since we assume relativistic quantum field theory to be correct, such a field must carry a Källén-Lehmann spectral density. But nothing tells us, that it has to be a single particle pole. Generally speaking that would not be the case. So assuming the existence of a Higgs boson field with an unknown spectral density, is the minimal assumption that one must make, in order to cure the problem of unitarity. Everything else is a stronger assumption, whether well or not so well motivated. Actually what this paper shows, is that even in the restricted class of renormalizable models with a single standard model Higgs, there is a broad choice of possibilities for a non-trivial spectrum. One should therefore accept this type of model as the generic form of the simplest Higgs mechanism, the single particle being a special case. This would also mean, that the LHC is not the right machine to study the mechanism of electroweak symmetry breaking. However it is a very good machine to rule out exotic models; it is potentially an almost perfect null-experiment for such a purpose. If the LHC finds no signal for new physics, not even for the Higgs particle, this would basically prove that the HEIDI models are the right class of models to describe electroweak symmetry breaking. This would provide an exceedingly strong physics argument for building a lepton collider. Such a collider would not need a very high energy; $300 \mathrm{GeV}$ would be more than enough. One needs however a high luminosity and a high precision on the beam energy. It is somewhat ironic, that this type of model was not discussed already in the 1960's, when the Higgs particle was introduced, as in this period much research was done on dispersion relations, which is a subject closely related to the Källén-Lehmann spectral density. Besides in the Higgs sector the idea of mixing a four-dimensional field with a high dimensional singlet in a renormalizable way works also for (abelian) vector-fields [17-19] or for right-handed neutrinos. Necessary is that the four-dimensional fields are singlets under the gauge group; otherwise renormalizability is lost.

Acknowledgements We thank Dr. O. Brein for discussions and a careful reading of the manuscript. This work was supported by the DFG within the Graduiertenkolleg "Physik an Hadron-Beschleunigern".

\section{References}

[1] ALEPH, DELPHI, L3 and OPAL Collaborations and the LEP Working Group for Higgs Boson Searches, Phys. Lett. B 565, 61 (2003).

[2] G. L. Kane, T. T. Wang, B. D. Nelson and L. T. Wang, Phys. Rev. D 71, 035006 (2005). 
[3] M. Drees, Phys. Rev. D 71, 115006 (2005).

[4] D. Hooper and T. Plehn, Phys. Rev. D 72, 115005 (2005).

[5] D. A. Demir, L. Solmaz and S. Solmaz, Phys. Rev. D 73, 016001 (2006).

[6] A. Hill and J. J. van der Bij, Phys. Rev. D 36, 3463 (1987).

[7] J.J. van der Bij, Phys. Lett. B 636, 56 (2006).

[8] J. J. van der Bij and S. Dilcher, Phys. Lett. B 638, 234 (2006).

[9] G. Källén, Helv. Phys. Acta 25, 417 (1952).

[10] H. Lehmann, Nuovo Cimento 11, 342 (1954).

[11] H. Georgi, Phys. Rev. Lett. 98, 221601 (2007).

[12] J. J. van der Bij and S. Dilcher, Phys. Lett. B 655, 183 (2007).

[13] W.D. Goldberger, M.B. Wise, Phys. Rev. D 65, 025011 (2001).

[14] S. Dilcher, Doktorarbeit Freiburg (2001).

[15] F. del Aguila, M. Pérez-Victoria, J. Santiago, JHEP0302, 051 (2003).

[16] C. de Rham, JHEP0801, 060 (2008).

[17] A. Ferroglia, A. Lorca and J.J. van der Bij, Ann. Phys. (Leipzig) 16, 563 (2007).

[18] B. Fuks, Qingjun Xu and J. J. van der Bij, Phys. Rev. D 78, 074016 (2008).

[19] N. V. Krasnikov, Mod. Phys. Lett. A25, 2313 (2010). 